\title{
SIGNAL RECONSTRUCTION VIA NOISE THROUGH A SYSTEM OF PARALLEL THRESHOLD NONLINEARITIES
}

\author{
Mark D. McDonnell and Derek Abbott
}

\author{
School of Electrical and Electronic Engineering \\ The University of Adelaide \\ Adelaide SA 5005 AUSTRALIA \\ email: mmcdonne@eleceng.adelaide.edu.au,dabbott@eleceng.adelaide.edu.au
}

\begin{abstract}
We present an analysis of the exploitation of noise for signal reconstruction by an array of nonlinear threshold-based devices. This phenomenon has been described as a form of stochastic resonance known as suprathreshold stochastic resonance. It occurs when all devices in an array of size $N$ have identical thresholds and are subject to independent additive noise. The original work showed that the mutual information between the input and output of the array has a maximum for a nonzero value of noise intensity, for a random input signal. In this paper we extend the results on this phenomenon to the case of Laplacian signal and noise probability densities, and show conditions exist under which it is optimal.
\end{abstract}

\section{INTRODUCTION}

The model we analyze can be considered as a method for analog to digital conversion in noisy conditions. It consists of an array of $N$ threshold devices (see Fig. 1) which receive the same input signal, $x$. The $n$-th device is subject to continuously valued iid additive noise, $\eta_{n}(n=1, . ., N)$ and the output from each, $y_{n}$, is unity if the input signal plus the noise is greater than its threshold, $\theta_{n}$, and zero otherwise. All outputs are summed to give the overall output signal, $y$. Hence, $y$ is a discrete signal taking on integer values from 0 to $N$ and can be considered as the number of devices that are currently "on" and thus

$$
y=\frac{1}{2} \sum_{n=1}^{N} \operatorname{sign}\left[x+\eta_{n}-\theta_{n}\right]+\frac{N}{2} .
$$

For the sake of generality, we assume that the input signal consists of uncorrelated samples of an iid random signal. Such an array can model various devices such as flash analog to digital converters subject to threshold noise, DIMUS (Digital Multibeam Steering) sonar arrays in the "on target"

This work was funded by the Australian Research Council. position [1] or a summing network of $N$ FitzHugh-Nagumo neurons [2].

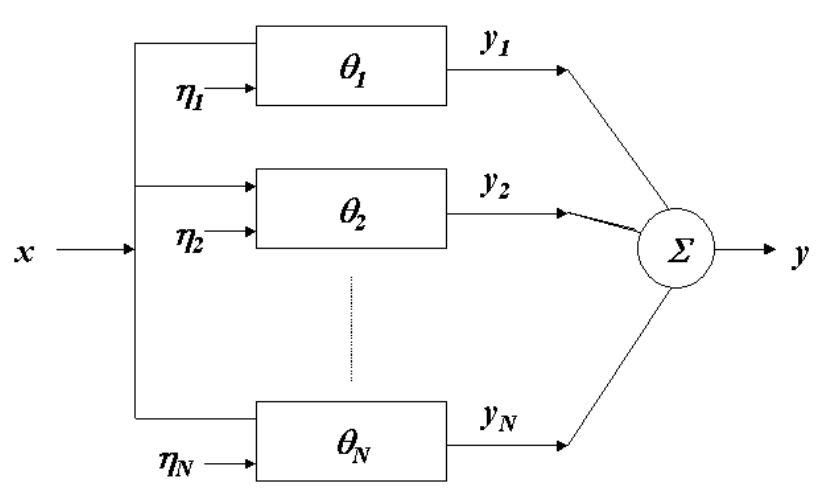

Fig. 1. Array of $N$ noisy threshold devices. Each device receives the same input signal, $x$, and is subject to independent additive noise, $\eta_{n}$. The output from device $n$ is zero if the sum of the signal and noise is greater than that device's threshold, $\theta_{n}$. The overall output, $y$, is the sum of the individual outputs.

Stocks has analyzed this system using Shannon information theory [3]. For the case of all thresholds set equal to the input signal mean, it was shown that the input-output mutual information has a maximum for nonzero noise. This phenomenon was termed Suprathreshold Stochastic Resonance (SSR). Conventional Stochastic Resonance (SR) occurs when the output of a nonlinear system is optimized by a nonzero value of internal or input noise (see, for example, [4]) and has been observed in many nonlinear systems including electronic circuits, ion channels, neurons and ring lasers (see [5] for an in-depth review). It has been proven that stochastic resonance only occurs in nonlinear systems. In many of these systems, the main nonlinearity is a Heavyside style threshold and it has been noted that for a system consisting of a single threshold, SR only occurs for sub- 
threshold signals. By contrast, SSR occurs for arbitrary signal magnitude, due to the presence of more than one threshold and therefore more than two output states. We note that SSR is similar to dithering in ADC's [6], with the difference being that dithering is the term used for the deliberate addition of noise in order to shape the output noise spectrum, whereas in the SSR model, the noise is assumed to be an inherent, unavoidable part of the system.

Several application areas exploiting the use of SSR have been suggested, including cochlear implant encoding based on SSR [7], and artifical insect-vision based motion detection devices [8]. Our research is focused on investigating these and other applications which could incorporate SSR to improve performance when compared to conventional means. As part of this investigation, we illustrate using a simple model that conditions exist where SSR can be optimal. In this paper we compare the output of the array of comparators in the SSR configuration to an array with distributed thresholds, which is the case for a flash analog to digital converter. Previous theoretical and numerical studies have used Gaussian or uniformly distributed signal and noise. Here we use Laplacian signal and noise, the reason being that the Laplacian probability density function provides computational speed advantages over the Gaussian, and yet unlike the uniform density has a realistic long tail.

\section{MATHEMATICAL MODELING}

The array of comparators gives a non-deterministic (except in the absence of noise) and lossy transform of an input signal to an output signal. Since the transform is not deterministic, probabilistic measures are appropriate since the output is uncertain given the input. The key function we need to be able to calculate is therefore the joint probability density between the input and output signals, $P_{x y}(x, n)$. All measures we could potentially use to describe the effects of the transform depend on this function. We commence by deriving a method of calculating $P_{x y}(x, n)$ for the general case of $N$ arbitrary thresholds, and then simplify to the SSR case of all thresholds equal to the signal mean.

Denote the probability density of the input signal as $P_{x}(x)$ and the probability mass function of the output as $P_{y}(n)$. Then we have, as a consequence of Bayes' theorem,

$$
P_{x y}(x, n)=P(n \mid x) P_{x}(x) .
$$

Integration of the the joint probability with respect to $x$ gives

$$
P_{y}(n)=\int_{-\infty}^{\infty} P(n \mid x) P_{x}(x) d x .
$$

We will assume knowledge of $P(x)$ and derive a method for calculating $P(n \mid x)$. Recall we assume that the noise density, $R(\eta)$, is iid at each comparator. Let $\hat{P}_{n}$ be the probability of device $n$ being "on" (that is, signal plus noise exceeding the threshold $\theta_{n}$ ), given the input signal $x$. Then

$$
\hat{P}_{n}=\int_{\theta_{n}-x}^{\infty} R(\eta) d \eta=1-F_{R}\left(\theta_{n}-x\right)
$$

where $F_{R}$ is the cumulative distribution function of the noise and $n=1, . ., N$. If the noise has an even probability density function then $\hat{P}_{n}=F_{R}\left(x-\theta_{n}\right)$.

In general, it is difficult to find an analytical expression for $P(n \mid x)$ and we will rely on numerics. Given a noise density and threshold value, $\hat{P}_{n}$ can be calculated exactly for any value of $x$ from (2). Assuming $\hat{P}_{n}$ has been calculated for desired values of $x$, a convenient way of numerically calculating the probabilities $P(n \mid x)$ for an array of size $N$ is as follows. Let $T_{n, k}$ denote the probability that $n$ of the devices $1, \ldots, k$ are "on," given $x$. Then let $T_{0,1}=1-\hat{P}_{1}$ and $T_{1,1}=\hat{P}_{1}$ and we have the recursive formula

$$
T_{n, k+1}=\hat{P}_{k+1} T_{n-1, k}+\left(1-\hat{P}_{k+1}\right) T_{n, k}
$$

where $k=1, . ., N-1, n=0, . ., k+1, T_{-1, k}=T_{k+1, k}=$ 0 and we have $P(n \mid x)$ given by $T_{n, N}$ [9]. For the particular case when the thresholds all have the same value, then each $\hat{P}_{n}$ has the same value $\hat{P}$ and, as noted by Stocks [3] we have the binomial distribution

$$
P(n \mid x)=C_{n}^{N} \hat{P}^{n}(1-\hat{P})^{N-n} \quad(0 \leq n \leq N) .
$$

Thus, for any arbitrary threshold settings and signal and noise probability distributions, $P(n \mid x)$ can be easily calculated numerically from (2) and (3).

The mutual information, $I$, between $x$ and $y$ is given by the entropy of the output, $H(y)$, less the conditional entropy of the output given the input, $H(y \mid x)$. As noted by Stocks [3], in our model $H(y \mid x)$ can be interpreted as the amount of encoded information about the input signal lost through the channel. Since the input to the array is continuously valued and the output is discretely valued, the channel can be considered to be semi-continuous. The mutual information through such a channel is given by

$$
\begin{aligned}
I= & -\sum_{n=0}^{N} P_{y}(n) \log _{2} P_{y}(n)- \\
& -\int_{-\infty}^{\infty} P(x) \sum_{n=0}^{N} P(n \mid x) \log _{2} P(n \mid x) d x
\end{aligned}
$$

and can therefore be calculated by numerical integration with the use of (1) and (3). 


\section{LAPLACIAN SIGNAL AND NOISE}

If the input signal $x$ has a Laplacian distribution with zero mean and variance $\sigma_{x}^{2}$ then

$$
P(x)=\frac{1}{\sqrt{2} \sigma_{x}} \exp \left(\frac{-\sqrt{2}|x|}{\sigma_{x}}\right)
$$

If the independent noise $\eta$ in each device has a Laplacian distribution with zero mean, and variance $\sigma_{r}^{2}$ then

$$
P(\eta)=\frac{1}{\sqrt{2} \sigma_{r}} \exp \left(\frac{-\sqrt{2}|\eta|}{\sigma_{r}}\right) .
$$

The cumulative distribution function of the noise is

$$
F_{R}(y)=0.5\left(1+\operatorname{sign}(y)\left(1-\exp \left(\frac{-\sqrt{2}|y|}{\sigma_{r}}\right)\right)\right)
$$

and thus

$$
\hat{P}_{n}= \begin{cases}0.5 \exp \left(\frac{-\sqrt{2}\left(\theta_{n}-x\right)}{\sigma_{r}}\right), & x \leq \theta_{n} \\ 1-0.5 \exp \left(\frac{\sqrt{2}\left(\theta_{n}-x\right)}{\sigma_{r}}\right), & x \geq \theta_{n}\end{cases}
$$

If we put $\theta_{n}=0 \forall n$ then

$$
\hat{P}= \begin{cases}0.5 \exp \left(\frac{\sqrt{2} x}{\sigma_{r}}\right), & x \leq 0 \\ 1-0.5 \exp \left(\frac{-\sqrt{2} x}{\sigma_{r}}\right), & x \geq 0\end{cases}
$$

\section{RESULTS}

\subsection{SSR results for Laplacian signal and noise}

Previous suprathreshold stochastic resonance results showed the existence of a maximum in the mutual information measure for a nonzero value of Gaussian or uniformly distributed noise when all thresholds are set to the signal mean. We obtain similar results for zero-mean iid Laplacian signal and noise by numerical integration of (5). This integration makes use of (1), (4), (6) and (7). The results obtained for various values of $N$ are shown in Fig. 2, where we have have let $\sigma_{x}=1$ and plotted the mutual information against increasing values of noise standard deviation, $\sigma_{r}$. For comparison, we have also shown the same plots for Gaussian signal and noise obtained in previous work [9].

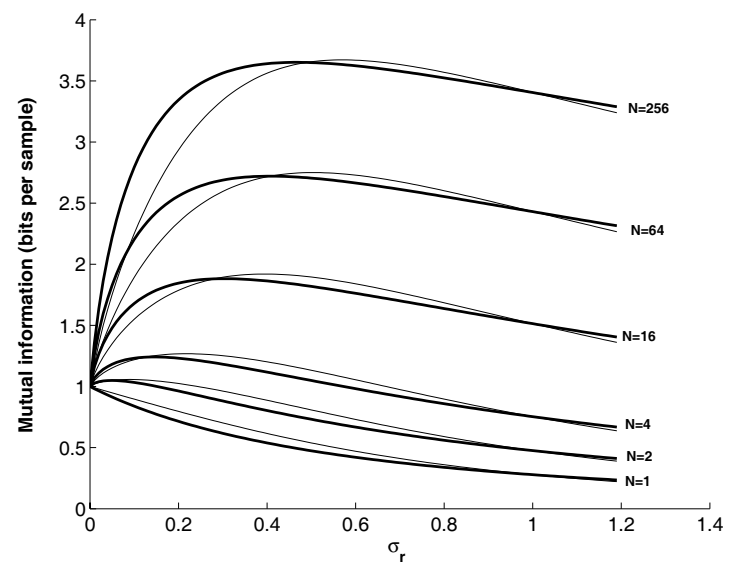

Fig. 2. Plot of mutual information against $\sigma_{r}$ for various values of $N$ and Laplacian signal and noise (thick solid line) and Gaussian signal and noise (thin solid line).

It is evident that as for Gaussian signal and noise, the SSR effect occurs for all values of $N$ other then $N=1$. As $N$ becomes large, the peak in the mutual information converges to a single value near $\sigma_{r}=0.5$. This is consistent with work by Hoch et al [10], who showed theoretically this same result for Gaussian signal and noise.

\subsection{Optimal thresholds for Laplacian signal and noise}

The key feature of SSR is a maximizing nonzero noise value for the case of all thresholds equal to the signal mean. However such a threshold setting does not necessarily maximize the mutual information. For example, in the noiseless case, with all thresholds equal to the signal mean the mutual information is only one bit per sample, whereas the mutual information is maximized when all output states are equally probable, and is equal to $\log _{2}(N+1)$ bits per sample. This is achieved by ensuring all output states are equally probable, that is, $P_{y}(n)=1 /(N+1)$ for all $n$. The optimal noiseless thresholds can be calculated from

$$
\int_{\theta_{n}}^{\theta_{n+1}} P(x) d x=\frac{1}{N+1} .
$$

For Laplacian signal and noise we obtain the optimal noiseless thresholds as

$$
\theta_{n}= \pm \frac{\sigma_{x}}{\sqrt{2}} \ln \left(\frac{2 n}{N+1}\right)
$$

where $n=1, . .,(N+1) / 2$.

We aim to find the optimal threshold settings for an arbitrary value of noise. This problem is equivalent to a noisy optimal quantization problem. For nontrivial $N$, even if we discretize the possible threshold values, the search space for 
the optimal thresholds increases combinatorially with $N$, and we must resort to heuristic or random search methods to obtain the optimal thresholds. We have trialed a genetic algorithm and simulated annealing for this problem, however best results have been achieved using a combination of gradient descent and a localized random search, where the initial trial solution for a certain value of noise, $\sigma_{r}$ is the optimal solution found for the previous, adjacent, value of noise, $\sigma_{r}-\Delta \sigma$. A plot showing the optimal thresholds obtained using this method for $N=3$ is shown in Fig. 3.

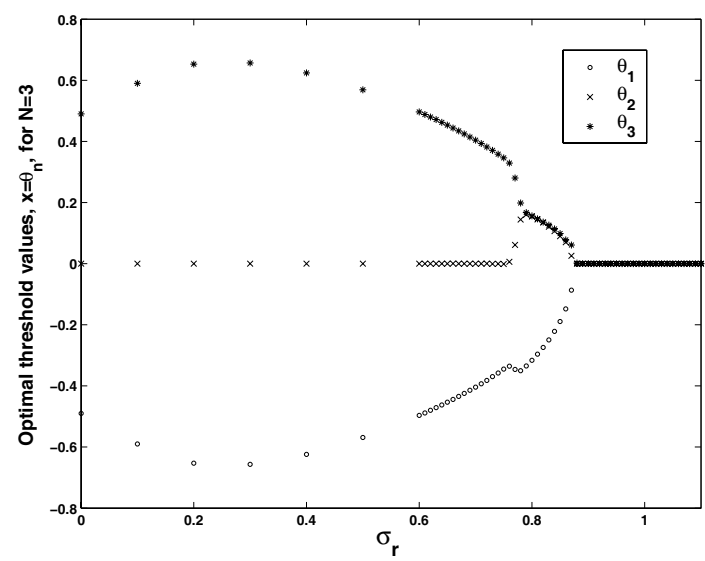

Fig. 3. Plot of optimal threshold values, $x=\theta_{n}$, against $\sigma_{r}$ for $N=3$ and Laplacian signal and noise.

For small values of noise the optimal thresholds are distributed well apart from each other. However, as the noise gets larger, the thresholds converge towards the same value and eventually, at about $\sigma_{r}=0.9$, the SSR configuration of all thresholds equal to the signal mean is optimal. There is also a region where it is not optimal to have thresholds distributed symmetrically about $x=0$, but to have two of the thresholds at the same value. We have found this asymmetry to consistently be the case for other signal and noise distributions, and measures other than mutual information, and are currently investigating the reasons for this [11].

\section{CONCLUSIONS}

The main results presented here is that the SSR effect occurs for Laplacian signal and noise and that, as for other distributions, the SSR configuration is optimal above a certain value of noise. This is significant for several reasons. Firstly, in the SR literature, it is often stated that the occurrence of SR in a single threshold is a suboptimal method of signal transfer. Here, we have shown that for the case of more than threshold device, that stochastic resonance can be optimal when the noise is sufficiently large. Secondly, the fact that SSR can be optimal has significance for potential applications based on the use of internal noise, since having all thresholds equal reduces the complexity of the system, compared to the case of requiring $N$ distinct thresholds, particularly when $N$ is large.

\section{REFERENCES}

[1] V.C. Anderson, "Digital array phasing," The Journal of the Acoustical Society of America, vol. 32, no. 7, pp. 867-870, 1960.

[2] N.G. Stocks and R. Mannella, "Generic noise enhanced coding in neuronal arrays," Physical Review E, vol. 64, pp. 030902(R), 2001.

[3] N.G. Stocks, "Suprathreshold stochastic resonance in multilevel threshold systems," Physical Review Letters, vol. 84, no. 11, pp. 2310-2313, 2000.

[4] H.C. Papadopoulos and G.W. Wornell, "A class of stochastic resonance systems for signal processing applications," in Proc. IEEE International Conference on Acoustics, Speech, and Signal Processing, 1996, pp. 1617-1620.

[5] L. Gammaitoni, P. Hänggi, and P. Jung, "Stochastic resonance," Reviews of Modern Physics, vol. 70, no. 1, pp. 223-287, 1998.

[6] R.A. Wannamaker, S.P. Lipshitz, and J. Vanderkooy, "Stochastic resonance as dithering," Physical Review E, vol. 61, no. 1, pp. 233-236, 2000.

[7] N.G. Stocks, D. Allingham, and R.P. Morse, “The application of suprathreshold stochastic resonance to cochlear implant coding," Fluctuation and Noise Letters, vol. 2, no. 3, pp. L169-L181, 2002.

[8] G.P. Harmer and D. Abbott, "Motion detection and stochastic resonance in noisy environments," Microelectronics Journal, vol. 32, no. 12, pp. 959-967, 2001.

[9] M. D. McDonnell, C. E. M. Pearce, and D. Abbott, "An analysis of noise enhanced information transmission in an array of comparators," Microelectronics Journal, vol. 33, no. 12, pp. 1079-1089, 2002.

[10] T. Hoch, G. Wenning, and K. Obermayer, "Adaptation using local information for maximizing the global cost," Neurocomputing, vol. 52-54, pp. 541-546, 2003.

[11] M.D. McDonnell, N.G. Stocks, C.E.M Pearce, and D. Abbott, "Maximising information transfer through nonlinear noisy devices," in Biomedical Applications of Micro and Nanoengineering, Dan V. Nicolau, Ed., 2002, vol. 4937 of Proceedings of SPIE, pp. 254-263. 